Geophysical Journal International

Supplementary material for the manuscript:

\title{
Multiple Graph Realizations Method: Improving the accuracy and the efficiency of the Shortest Path Method through random sampling.
}

Petros Bogiatzis*1 ${ }^{1}$, Catherine A. Rychert ${ }^{1}$ and Nicholas Harmon ${ }^{1}$

${ }^{1}$ Ocean and Earth Science, National Oceanography Centre Southampton, University of Southampton, Southampton, UK.

Corresponding author: Petros Bogiatzis (P.Bogiatzis@ soton.ac.uk)

\section{Contents of this file}

Figures S1 to S6

Table S1 

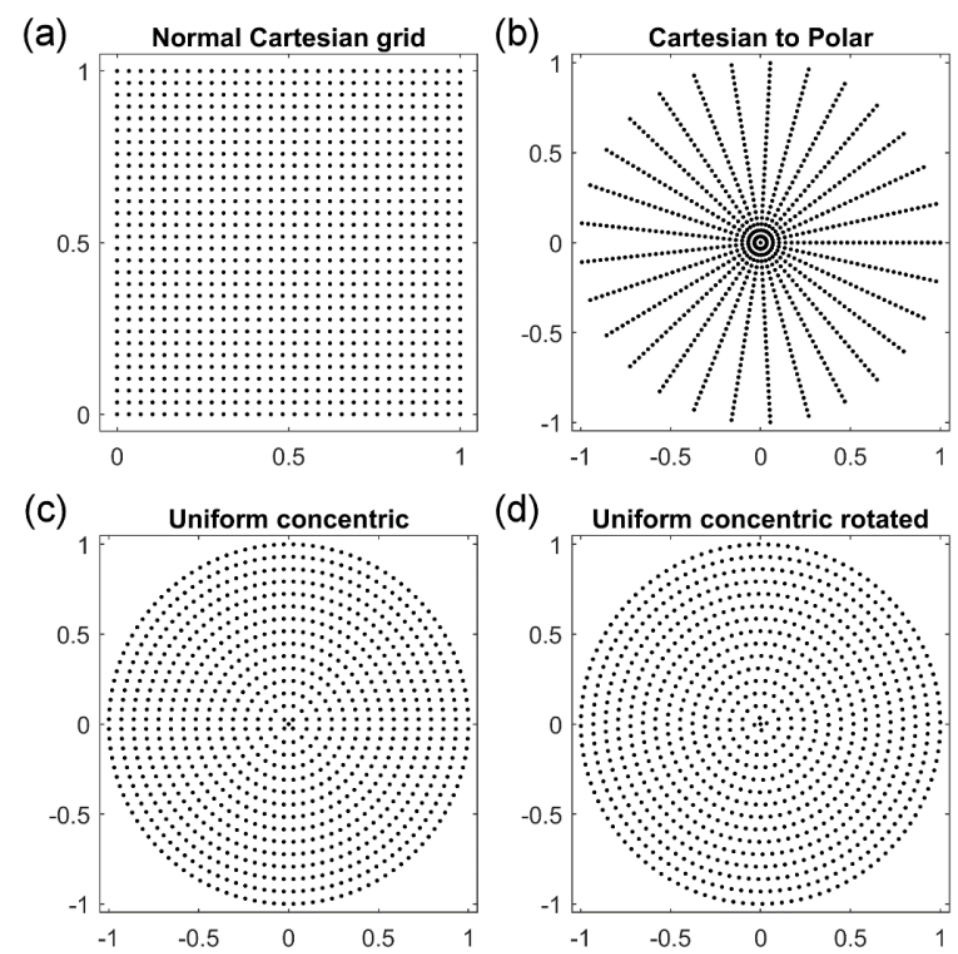

Figure S1: (a) Normal cartesian grid and two different maps. (b) Cartesian to polar mapping, (c) concentric mapping and (d) concentric mapping after rotation. 


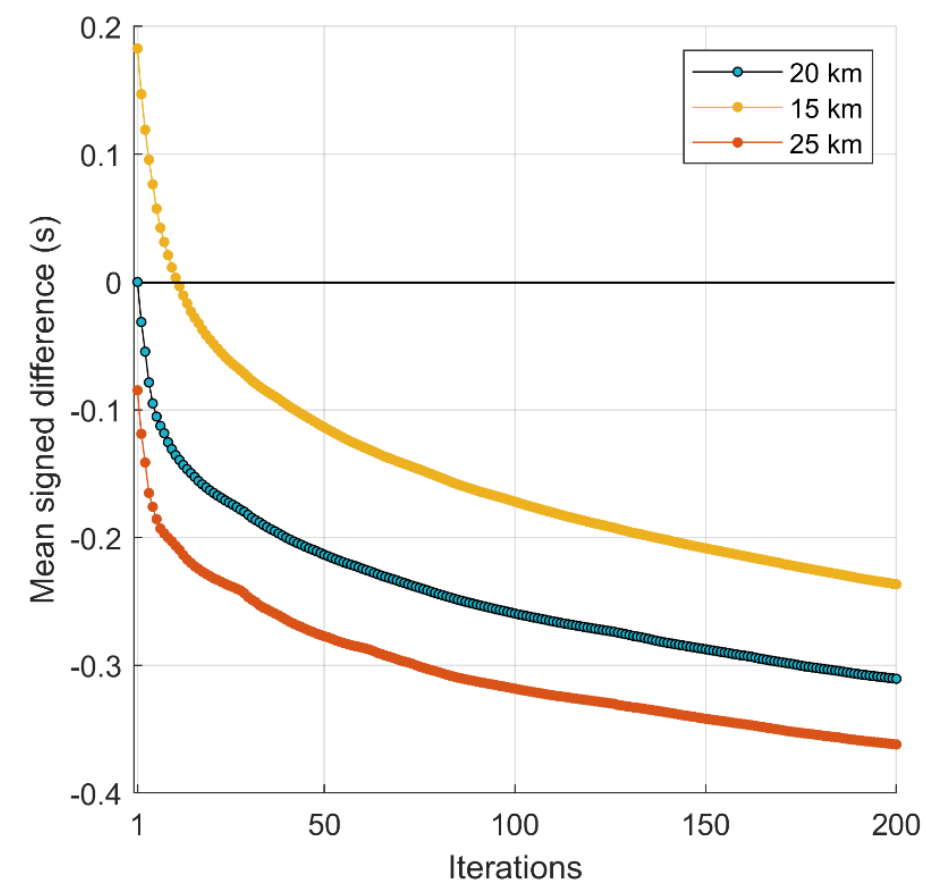

Figure S2: Application of the MGR method in the subduction model described in main text, using three different connectivity distance thresholds. The reference graph is the same used in main text which uses connectivity distance of $20 \mathrm{~km}$. When the connectivity distance is 15 $\mathrm{km}$ the computational time for 200 iterations is $\sim 7 \%$ smaller, while in the case of $25 \mathrm{~km}$ it increases by $15 \%$ with respect to the connectivity distance of $20 \mathrm{~km}$. 

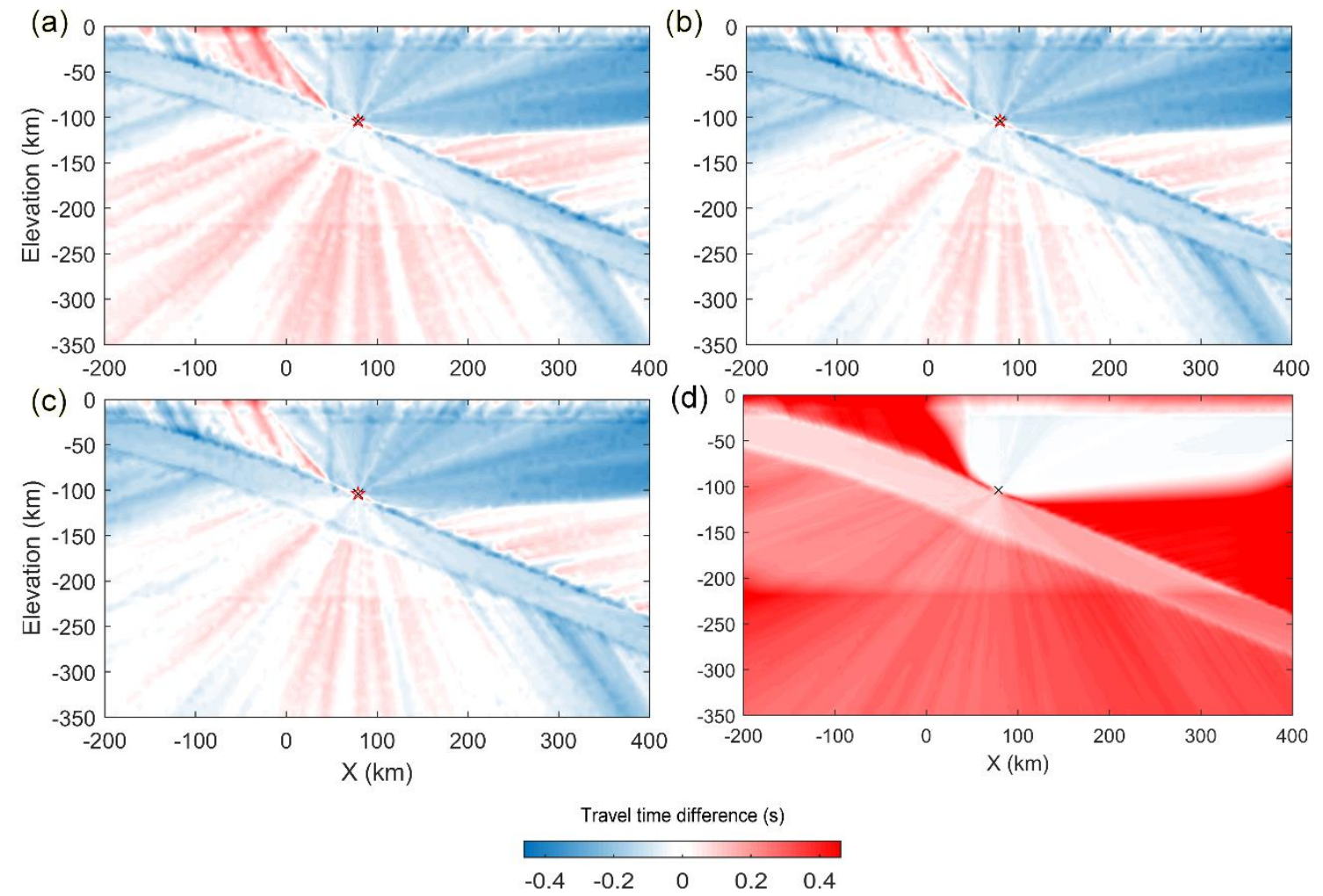

Figure S3: Travel time difference between the FMM method for spacing of (a) $1000 \mathrm{~m}$, (b) $100 \mathrm{~m}$ and (c) $50 \mathrm{~m}$, with a single application of the shortest path method using the initial graph. The star shows the location of the source. The results are interpolated in the reference grid. The average CPU time for each application of the FMM method is (a) $0.28 \mathrm{~s}$, (b) $12 \mathrm{~s}$ and (c) $65 \mathrm{~s}$ respectively. (d) Travel time difference between FMM for spacing of 100m and the MGR method after 200 iterations. 
(a)

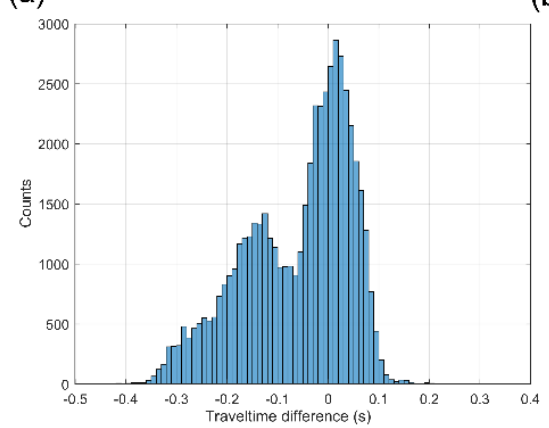

(d)

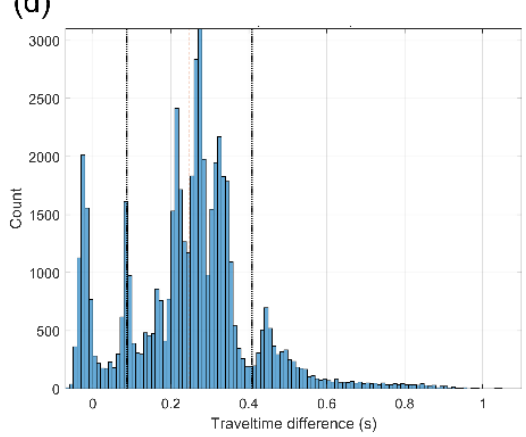

(b)

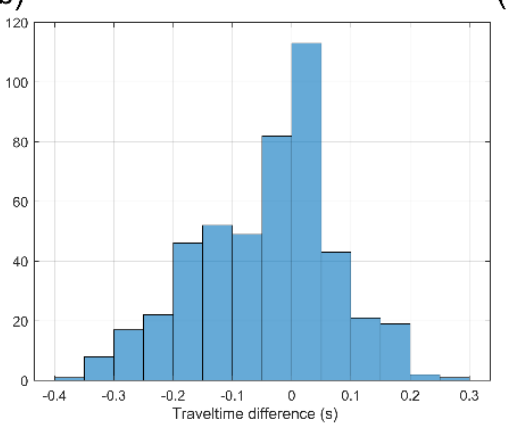

(c)

(e)

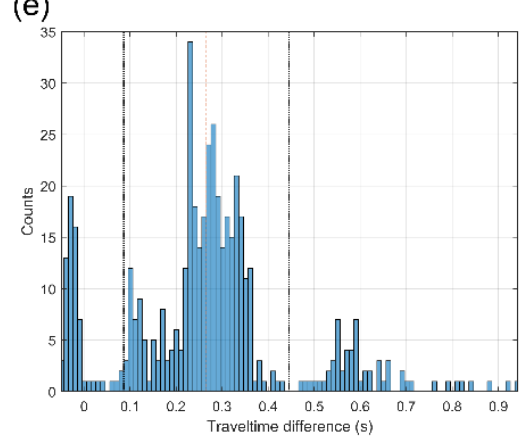

Figure S4: Histogram of travel time differences between the (a) FMM for spacing of $100 \mathrm{~m}$ and the single application of the SPM using the initial graph. (b) Likewise, but for the SOFI2D result and the SPM using the initial graph (c) likewise but for the MGR method and the SPM using the initial graph. (d) Histogram of the travel time differences between the FMM and the MGR method after 200 iterations. (e) The same but for the SOFI2D and the MGR method. The dashed lines show the mean difference and the dotted lines mark the distance of one standard deviation from the mean. 

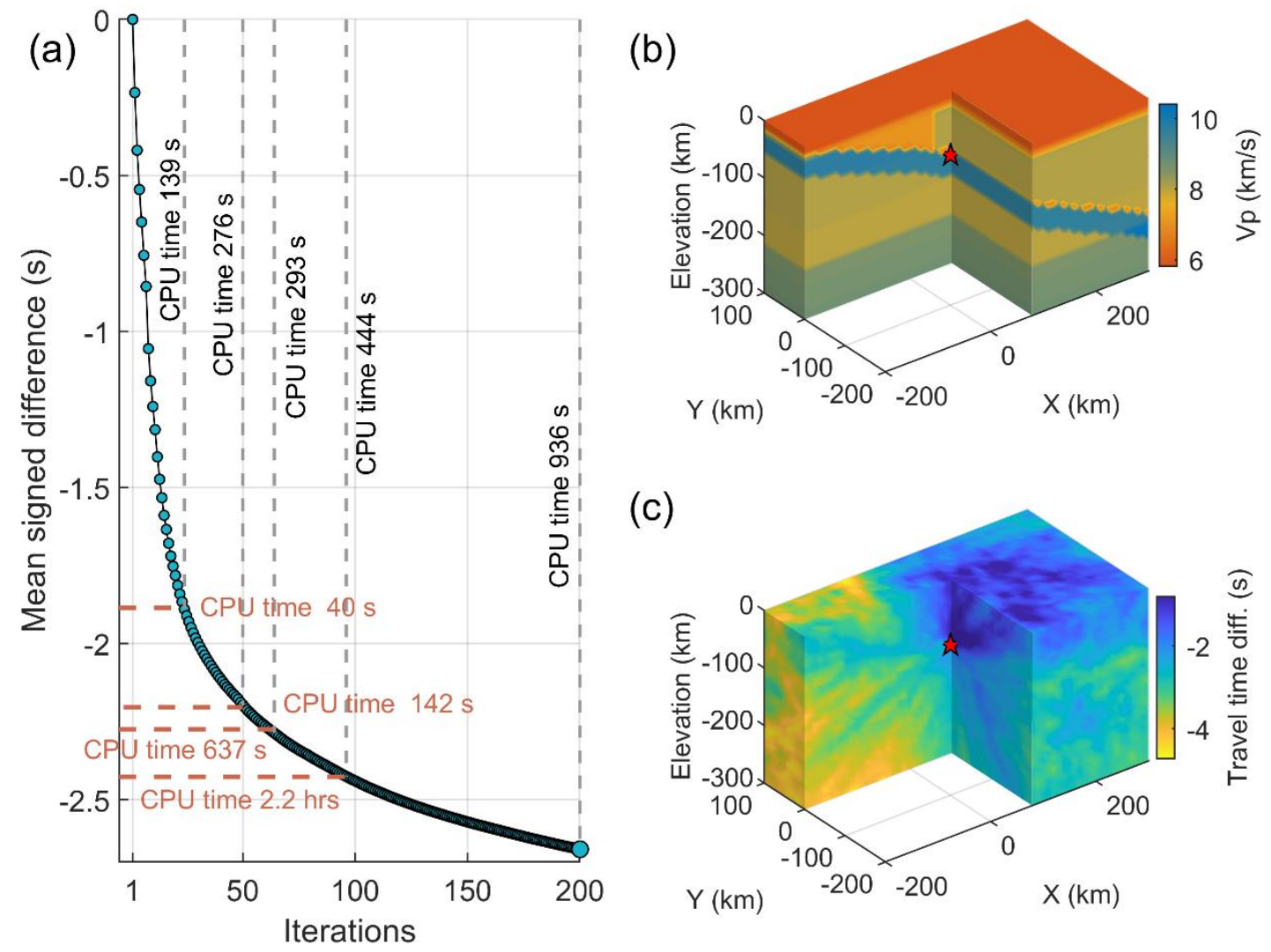

Figure S5: Results of the MGR method for the heterogeneous 3-D model that imitates a subduction zone (a) The Mean signed difference from the initial graph as a function of the iteration number. Vertical dashed lines indicate the computation time up of the MGR method at different iterations. The horizontal dashed lines show the corresponding computation time needed for achieving similar improvement in travel times by applying the shortest path method only once, in a denser graph. From the top to the bottom, for average node distance of $10 \mathrm{~km}$, $8 \mathrm{~km}, 6 \mathrm{~km}$ and $4.4 \mathrm{~km}$. (b) The velocity model used for the computations. The model is constructed from the 2-D model (Figure 6) that is described in the main text by extending (repeating) the structure along the strike (y direction) of the 2-D model (Figure S2b). The source location is indicated with the star (b) Travel time Improvement (s) using the MGR method, after 200 iterations. 
Table S1: Summary of results of MGR method against single runs with increasing grid density, for the 3-D case.

\begin{tabular}{c|cccccc}
\hline Method & $\begin{array}{c}\text { Grid } \\
\text { spacing } \\
(\mathbf{k m})\end{array}$ & Nodes & Connections & $\begin{array}{c}\text { Adjacency } \\
\text { matrix size } \\
(\mathbf{M B})\end{array}$ & $\begin{array}{c}\text { Average } \\
\text { travel time } \\
\text { reduction } \\
(\mathbf{s})\end{array}$ & $\begin{array}{c}\text { CPU } \\
\text { time } \\
(\mathbf{s})\end{array}$ \\
\hline MGR 200 & 15 & 28,506 & 247,213 & 4 & 2.659 & 936 \\
\hline iterations & 10 & 38,069 & $2,242,452$ & 35 & 1.885 & 40 \\
& 8 & 79,731 & $7,812,820$ & 120 & 2.204 & 142 \\
Single run & 6 & 151,661 & $40,544,895$ & 621 & 2.275 & 637 \\
& 4.4 & 874,929 & $244,622,684$ & 3,739 & 2.427 & 8,009 \\
\hline
\end{tabular}


(a)

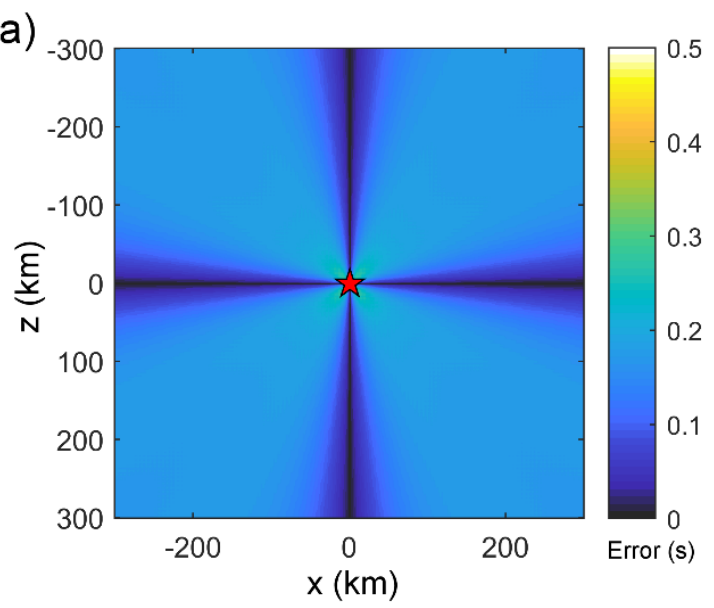

(b)

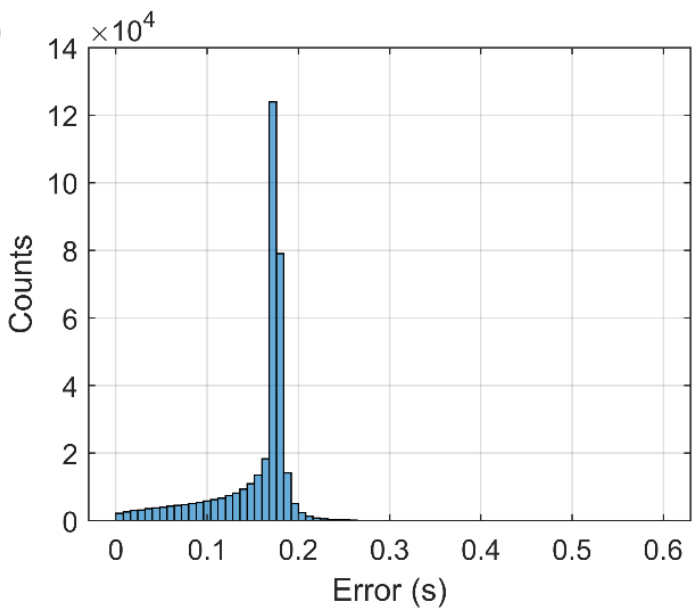

Figure S6: Error analysis of the second order FMM method used in this work against the analytical solution for the same constant velocity model as with Figure 4 of the manuscript. (a) Spatial distribution of errors in (s). Source location is shown as a star. (b) Histogram of errors. The FMM grid is the same with the regular grid used for the SPM, of Figure 4a. 